\title{
Increased tumor cell proliferation in mantle cell lymphoma is associated with elevated insulin-like growth factor 2 mRNA-binding protein 3 expression
}

Elena M Hartmann ${ }^{1}$, Sílvia Beà ${ }^{2}$, Alba Navarro ${ }^{2}$, Vanessa Trapp ${ }^{1}$, Elías Campo ${ }^{2}$, German $\mathrm{Ott}^{3}$ and Andreas Rosenwald ${ }^{1}$

${ }^{1}$ Institute of Pathology, University of Würzburg, Würzburg, Germany; ${ }^{2}$ Hematopathology Section, Laboratory of Molecular Pathology, Hospital Clínic i Provincial de Barcelona, Universitat de Barcelona, Barcelona, Spain and ${ }^{3}$ Department of Clinical Pathology, Robert-Bosch-Krankenhaus, and Dr Margarete Fischer-Bosch Institute of Clinical Pharmacology, Stuttgart, Germany

Mantle cell lymphoma is an aggressive, non-curable B-cell lymphoma, characterized by the translocation $t(11 ; 14)(q 13 ; q 32)$ involving $C C N D 1$ and a high number of additional genetic alterations. Chromosomal gains of $7 p$ are frequent in mantle cell lymphoma, with insulin-like growth factor II mRNA-binding protein 3 (IGF2BP3 aka IMP3) being the most upregulated gene in this region. IGF2BP3 is a member of the IGF II mRNA-BP family, and increased IGF2BP3 expression is associated with an aggressive behavior in many malignant tumors. We here analyze selected genes related to IGF signaling in gene expression and genomic array data of 8 mantle cell lymphoma cell lines and 12 primary mantle cell lymphomas and study IGF2BP3 protein expression in 172 wellcharacterized primary mantle cell lymphomas by immunohistochemistry. The majority of mantle cell lymphoma cell lines and primary cases showed elevated IGF2BP3 mRNA expression and a subset also expressed the IGF1 and IGF2 receptors. On the protein level, 66 of 172 primary mantle cell lymphomas showed IGF2BP3 expression in $>\mathbf{5 0} \%$ of tumor cells, and strong IGF2BP3 protein expression was highly associated with increased proliferation as measured by the $\mathrm{Ki}-67$ index, but not with overall survival of mantle cell lymphoma patients. Only a subset of mantle cell lymphomas with marked IGF2BP3 expression had an underlying chromosomal gain in 7p, suggesting that additional mechanisms are involved in the upregulation of IGF2BP3 in mantle cell lymphoma. In seven paired mantle cell lymphoma samples, IGF2BP3 protein expression remained constant between primary diagnosis and relapse. Increased IGF2BP3 expression and, potentially, enhanced IGF signaling may contribute proproliferative stimuli in the evolution of mantle cell lymphoma tumor cells. Modern Pathology (2012) 25, 1227-1235; doi:10.1038/modpathol.2012.84; published online 4 May 2012

Keywords: IGF2BP3; mantle cell lymphoma; proliferation; 7p genomic status

Mantle cell lymphoma is a non-curable and aggressive subtype of B-cell non-Hodgkin lymphoma with a median survival of 3-5 years. Almost all mantle cell lymphoma tumors carry the characteristic chromosomal translocation $\mathrm{t}(11 ; 14)(\mathrm{q} 13 ; \mathrm{q} 32)$ involving CCND1. However, another important biological feature of this lymphoma is the high number of

Correspondence: Dr A Rosenwald, MD, Institute of Pathology, University of Würzburg, Josef-Schneider-Str.2, 97080 Würzburg, Germany.

E-mail: Rosenwald@mail.uni-wuerzburg.de

Received 29 January 2012; revised 12 March 2012; accepted 13 March 2012; published online 4 May 2012 additional alterations in the tumor genomes that frequently target the regulation of cell cycle control genes and the DNA-damage response pathway. ${ }^{1,2}$ In recent years, several studies highlighted biological and prognostic implications of genetic gains and losses in the most commonly altered regions, leading to the hypothesis that variably affected target genes and associated pathways contribute to the highly divergent clinical behavior of mantle cell lymphoma ranging from very aggressive forms to more indolent clinical courses. ${ }^{2}$

A gain of the chromosomal region $7 p$ is frequent in mantle cell lymphoma ${ }^{2-5}$ and is associated with a 
blastoid morphology. ${ }^{4}$ A previous study using combined single-nucleotide polymorphism (SNP) and mRNA expression profiling directed our attention to the insulin-like growth factor (IGF) signaling pathway, as the IGF2 mRNA-binding protein 3 (IGF2BP3 aka IMP3) was the most strongly upregulated gene in cases harboring this alteration. ${ }^{5}$ Moreover, IGF2BP3 has been described to belong to the 'mantle cell lymphoma gene expression signature', ${ }^{6}$ and IGF2BP3 mRNA expression was found to be predictive of outcome in mantle cell lymphoma in an in silico analysis of gene expression data. ${ }^{7}$

Together with IGF2BP1 and 2 (aka IMP1 and 2), IGF2BP3 belongs to the family of IGF2-mRNA BPs that affect RNA stability and influence cell growth and migration during embryonic development. ${ }^{8}$ These proteins are part of the large group of regulators of the IGF pathway, which has a key role in the orchestration of cellular proliferation. ${ }^{9,10}$ The IGF pathway includes the ligands IGF1 and IGF2 that can bind to their receptors IGF1R and 2R, the latter of which is believed to function as a decoy receptor. IGF1 and 2 are regulated by a number of binding proteins ${ }^{10}$ and also by mRNA BPs such as IGF2BP3. Originally, IGF2BP3 was found to be overexpressed in pancreatic cancer. ${ }^{11}$ More recently, increased IGF2BP3 protein expression has been reported in a variety of solid tumors and was found to be associated with a higher histological grade, ${ }^{12}$ progression or metastasis ${ }^{13,14}$ and with an unfavorable outcome. ${ }^{15-18}$ These data indicate that IGF2BP3 may promote tumor cell proliferation and progression by enhancing signaling via the IGF pathway. In lymphoid cells, IGF2BP3 is physiologically expressed in normal germinal center $\mathrm{B}$ cells and an initial study described variable IGF2BP3 protein expression in different types of malignant lymphomas, particularly in those with an association to a germinal center B-cell phenotype. ${ }^{19}$ The aims of this study were to characterize the expression of members of the IGF signaling pathway, in particular IGF2BP3, in mantle cell lymphoma, and to determine a potential association with the aggressive biological behavior of this lymphoma.

\section{Materials and methods}

\section{Patients and Specimens}

In this study, IGF2BP3 expression was investigated in 8 mantle cell lymphoma cell lines (JEKO1, MAVER, MINO, UPN1, REC1, HBL2, GRANTA519 and JVM2) and 172 primary mantle cell lymphoma cases from the Institute of Pathology in Würzburg, Germany, and the Hospital Clinic of Barcelona, Spain. The pilot series consisted of whole tissue sections from 66 consecutive, formalin-fixed and paraffin-embedded mantle cell lymphoma cases (lymph nodes), selected from the files of the Institute of Pathology, Würzburg, between the years 2008 and 2010. The second series comprised 106 mantle cell lymphoma specimens with available clinical data. Of these, 45 mantle cell lymphomas were studied on whole tissue sections and 61 mantle cell lymphomas could be investigated in a tissue microarray (TMA) format. A total of 34 mantle cell lymphomas of the second series had information available on the genetic status of chromosome 7 as determined in some of our previous studies.,5,20 Histology of all primary cases was reviewed by expert hematopathologists, and the diagnosis of mantle cell lymphoma was established according to current criteria of the World Health Organization classification. ${ }^{21}$ All cases were CCND1 positive. In addition, we were able to investigate seven paired mantle cell lymphoma samples at the time of primary diagnosis and at relapse. For comparison, we investigated IGF2BP3 protein expression in a series of 20 small lymphocytic lymphomas from the files of the Institute of Pathology in Würzburg, Germany. Ethics approval for this study was granted by the Local Ethics Committees.

\section{Gene Expression and SNP Array Studies}

Gene expression studies of the eight mantle cell lymphoma cell lines were performed using HG U133 Plus 2.0. oligonucleotide arrays from Affymetrix (Affymetrix, Santa Clara, CA, USA). The samples were processed following the Affymetrix Expression analysis technical manual (available at http:// www.Affymetrix.com). Genome-wide analysis of chromosomal copy number alterations were performed using SNP arrays from Affymetrix (500K SNP-A) following standard protocols, and the data were analyzed by visual inspection according to previously reported methods., ${ }^{5,20}$ Additional gene expression and copy number data were derived from previous publications. ${ }^{4,5}$

\section{Immunohistochemistry}

Immunohistochemistry for IGF2BP3 was performed on $3-\mu \mathrm{m}$ paraffin-embedded tissue sections using a mouse monoclonal antibody (clone 69.1, Dako; 1:100 dilution in Dako Antibody Diluent; DAKO, Hamburg, Germany) according to the manufacturer's instructions and according to the standard immunohistochemical protocols. In brief, after deparaffinization and antigen retrieval by pressure cooking, the samples were incubated with the IGF2BP3 antibody (1:100) for $1 \mathrm{~h}$, followed by an incubation with the secondary antibody (link antibody, DAKO HRP Kit, ready to use) and HRP enzyme for $30 \mathrm{~min}$ each and visualization with the DAB system (all reagents from the Advance HRP Kit from DAKO). After each incubation, slides were washed three times for $8 \mathrm{~min}$ each in phosphate buffered saline. Normal tonsil specimens were used as positive controls. 
IGF2BP3 immunohistochemistry was evaluated by two different analysis algorithms adopted with modifications from King et al ${ }^{19}$ and Schaeffer et al. ${ }^{15}$ All cases were reviewed by two independent investigators. In the first scoring approach (adopted from King et $a l^{19}$ ), the percentage of positively stained tumor cells in each sample was evaluated. IGF2BP3 staining was predominantly cytoplasmic. Specifically, cases with $\geq 50 \%$ of tumor cells labeled by the IGF2BP3 antibody at any intensity were considered 'positive', cases with 10-49\% of tumor cells stained at any intensity were considered 'intermediate' and those with $<10 \%$ positive tumor cells were regarded 'negative'. In the second scoring approach (adopted from Schaeffer et $a l^{15}$ ), the intensity of IGF2BP3 staining was graded as negative, $1+$ (weak), $2+$ (moderate) or $3+$ (strong), and the percentage of positively stained tumor cells in each case was scored as $1+(<10 \%$ positive cells), $2+(10-49 \%), 3+(50-80 \%)$ and $4+(>80 \%)$. The final score was calculated by multiplying these two variables, with a score of $0-3$ considered as 'negative', 4-7 as 'and >8 as 'positive'. Mantle cell lymphoma cases without any positive cell for IGF2BP3 were excluded from the analysis.

Standard immunohistochemistry for CD20, CD5, Ki-67 and cyclin D1 was performed using automated standard protocols. Ki-67 scoring was accomplished by two independent pathologists defining four proliferation groups (5-20, 21-40, 41-60 and $>60 \%$ of tumor cells). ${ }^{22}$

\section{Tissue Microarrays (TMAs)}

The TMAs were constructed from archived conventional formalin-fixed paraffin-embedded tissue blocks using a Beecher Instruments manual tissue arrayer (Beecher Instruments, Sun Prairie, WI, USA). Representative tumor areas were chosen by two expert pathologists and five cores from each sample were transferred into the recipient block.

\section{Statistical Analysis}

Statistical analysis was performed using the $\chi^{2}$ test and ANOVA. Survival was estimated with the Kaplan-Meier method and compared by log-rank tests. $P$-values $<0.05$ were considered statistically significant. Statistical tests were performed using SPSS v14 software (SPSS, Chicago, IL, USA).

\section{Results}

Gene Expression of IGF2BP3 and Other Members of the IGF Signaling Pathway in Mantle Cell Lymphoma Cell Lines and Primary Mantle Cell Lymphomas

Our gene expression experiments in eight mantle cell lymphoma cell lines demonstrated an overall high expression level of IGF2BP3 mRNA in mantle cell lymphomas, however, with a 34-fold expression difference across the cell lines (Figure 1a). The highest expression levels of IGF2BP3 mRNA were observed in HBL2, UPN1, JEKO1 and MINO. Interestingly, parallel SNP array analysis revealed that JEKO1 and MINO both harbor a gain of the chromosomal region encompassing the IGF2BP3 locus on chromosome 7p. In addition, HBL2 showed a copy number neutral loss of heterozygosity of large regions of chromosome 7 . Figure 1 also displays mRNA expression levels of other members of the $I G F 2$-mRNA BP family as well as of $I G F 1 / 2, I G F 1 R$ and IGF2R. IGF2BP1 and 2 mRNAs were expressed at very low levels in all cell lines, as were IGF1 and 2 mRNAs. IGF1R mRNA showed a low, but variable expression (8.5-fold difference) across the mantle cell lymphoma cell lines, with the highest expression in JEKO1, MINO and HBL2. IGF2R also showed a low and differential expression in the cell lines, albeit less pronounced (fivefold expression difference) compared with IGF1R.

Primary mantle cell lymphoma cases from our previous study ${ }^{5}$ showed a more variable expression of IGF2BP3 mRNA and, again, the two mantle cell lymphomas with the highest IGF2BP3 gene expression showed a gain of the IGF2BP3 locus in $7 \mathrm{p}$ (Figure 1b). IGF2BP2 mRNA was expressed at very low levels in the primary cases, and no expression of $I G F 2 B P 1$ was observed. IGF1 was expressed at relatively low levels, and no expression was observed for IGF2. Both IGF1R and IGF2R showed a low and differential expression of around fourfold.

Taken together, among the investigated genes belonging to the IGF signaling pathway, only $I G F 2 B P 3$ appears to be significantly expressed in mantle cell lymphoma at the mRNA level.

\section{IGF2BP3 Protein Expression in Primary Mantle Cell Lymphomas}

We next investigated IGF2BP3 expression on the protein level by immunohistochemistry in a large series of 172 primary mantle cell lymphoma cases. The basic clinical and pathological features of the pilot series of 66 mantle cell lymphoma as well as the second series of 106 mantle cell lymphoma with available outcome data are summarized in Tables 1 and 2. The patients in the second series received heterogeneous treatments, including CHOP or CHOP-like therapies with or without anti-CD20 antibody as well as intensified treatment approaches (ie, HyperCVAD).

Figure 2 shows representative immunohistochemical staining results for IGF2BP3 in the pilot series with high, intermediate and low expression levels demonstrating a high variation of IGF2BP3 protein expression levels in mantle cell lymphoma. In tonsils, used as positive controls, IGF2BP3 protein was expressed in the cytoplasm of normal germinal center B cells, as reported previously. ${ }^{19}$ Whenever 

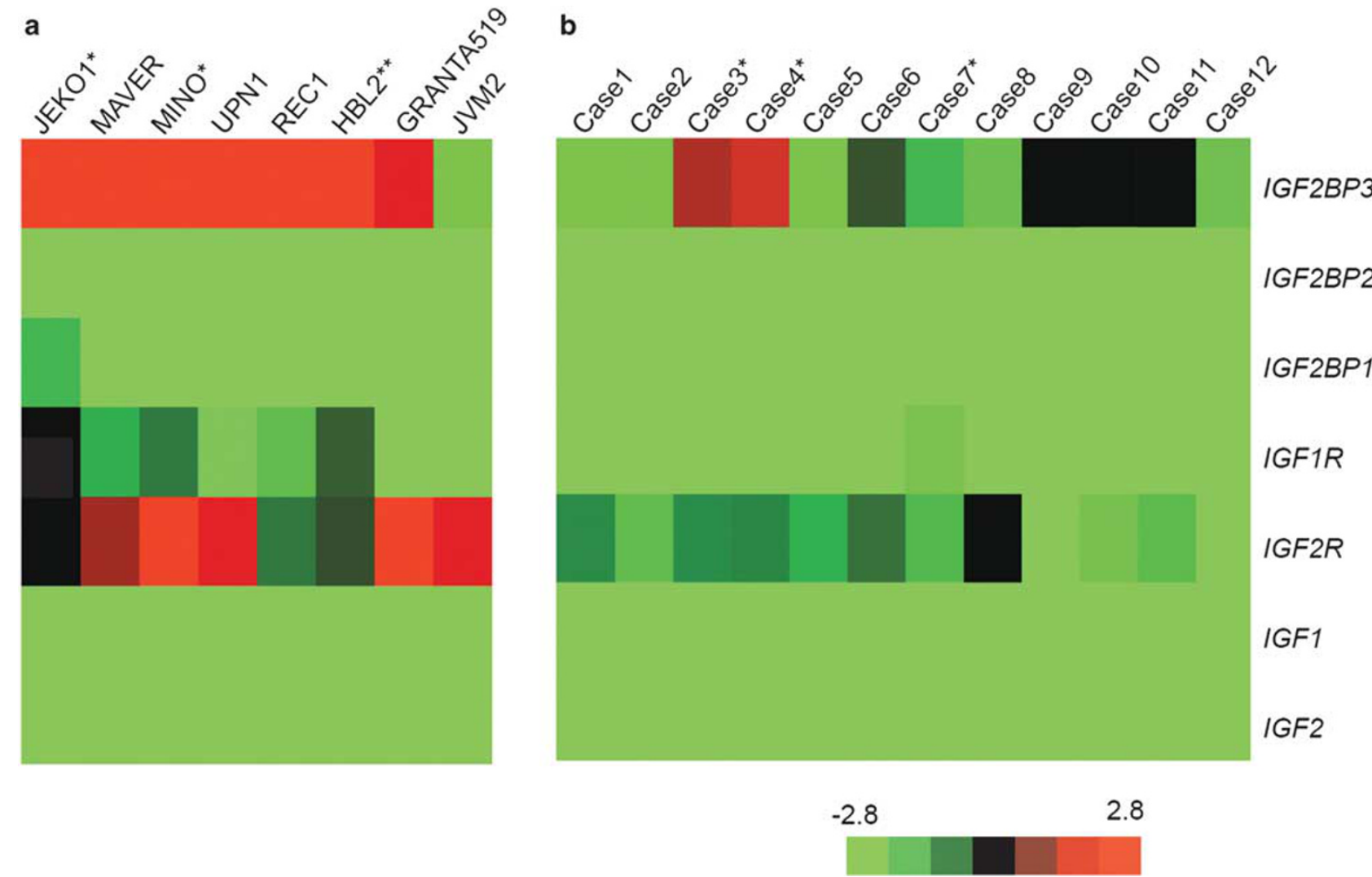

Figure 1 Relative mRNA expression of selected genes related to IGF signaling in mantle cell lymphoma. Heatmaps depicting the relative mRNA expression levels of IMP1, 2 and 3, IGF 1 and 2 as well as IGF1R and 2R in (a) 8 mantle cell lymphoma cell lines and (b) 12 primary samples from a previous study ${ }^{5}$ measured by microarray analysis. The relative mRNA expression levels are represented by a color scale from green, indicating low expression, to red, indicating high expression. One asterisk indicates gain of chromosome 7 or $7 \mathrm{p}$, and two asterisks indicate copy neutral loss of heterozygosity.

Table 1 Basic clinical and pathological features of primary mantle cell lymphomas in the pilot series (whole slides; $n=66$ )

\begin{tabular}{lc} 
Sex & \\
Male & $44(67 \%)$ \\
Female & $21(32 \%)$ \\
NA & $1(1 \%)$ \\
Morphology & \\
Classic & $55(83 \%)$ \\
Blastoid & $11(17 \%)$ \\
& \\
Age at diagnosis (years) & \\
Mean & 70 \\
Median & 71 \\
Range & $44-93$ \\
Ki67 (\%) & \\
Mean & \\
Median & 32 \\
Range & 30 \\
\hline
\end{tabular}

Abbreviation: NA, not available.

mantle cell lymphoma infiltrates contained residual germinal center B cells, these stained positively for IGF2BP3 serving as an internal control. Interestingly, IGF2BP3 expression in some mantle cell
Table 2 Basic clinical and pathological features of primary mantle cell lymphomas in the second series (45 whole slides+61 TMA; total $n=106$ )

\begin{tabular}{lc}
\hline Sex & \\
Male & $77(72 \%)$ \\
Female & $23(22 \%)$ \\
NA & $6(6 \%)$ \\
& \\
Morphology & $80(75 \%)$ \\
Classic & $24(23 \%)$ \\
Blastoid & $2(2 \%)$ \\
NA & \\
Age at diagnosis (years) & \\
Mean & 66 \\
Median & 67 \\
Range & $29-85$ \\
Ki67 (\%) & \\
Mean & 39 \\
Median & 37.5 \\
Range & $5-92$ \\
Survival (months) & \\
Mean & \\
Median & 38.4 \\
Range & 25.8 \\
\end{tabular}

Abbreviations: NA, not available; TMA, tissue microarray. 
a

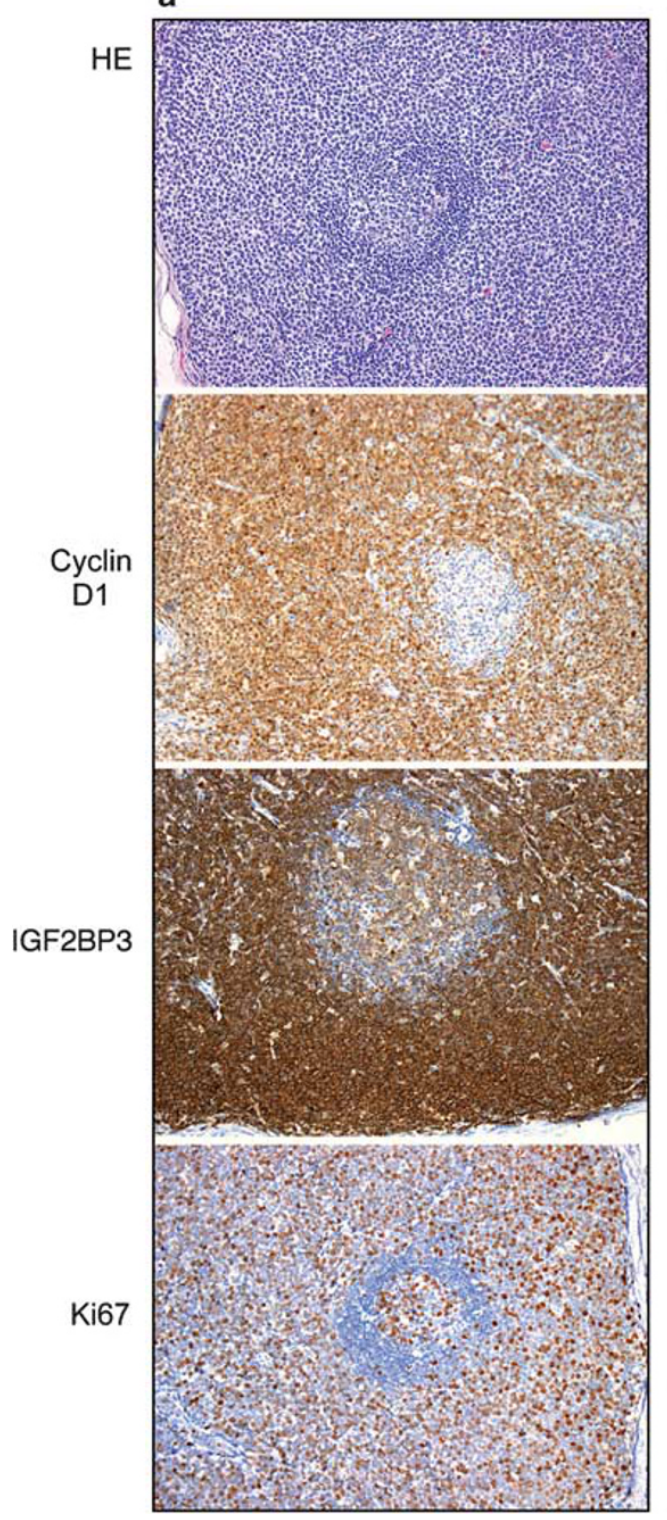

b

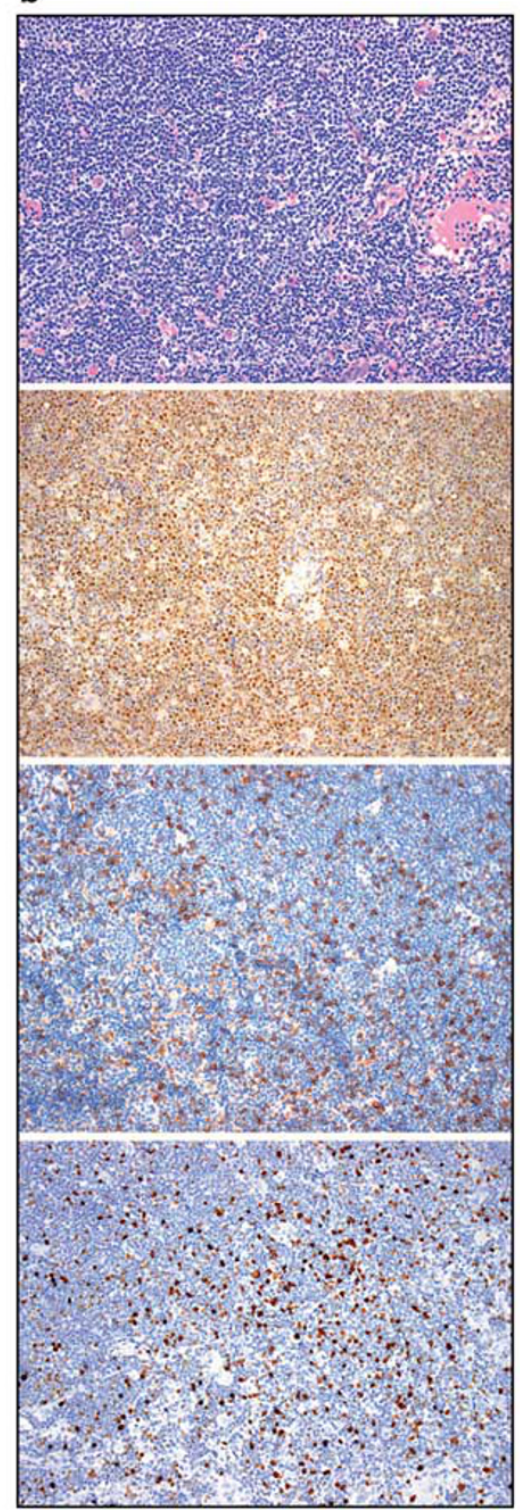

c

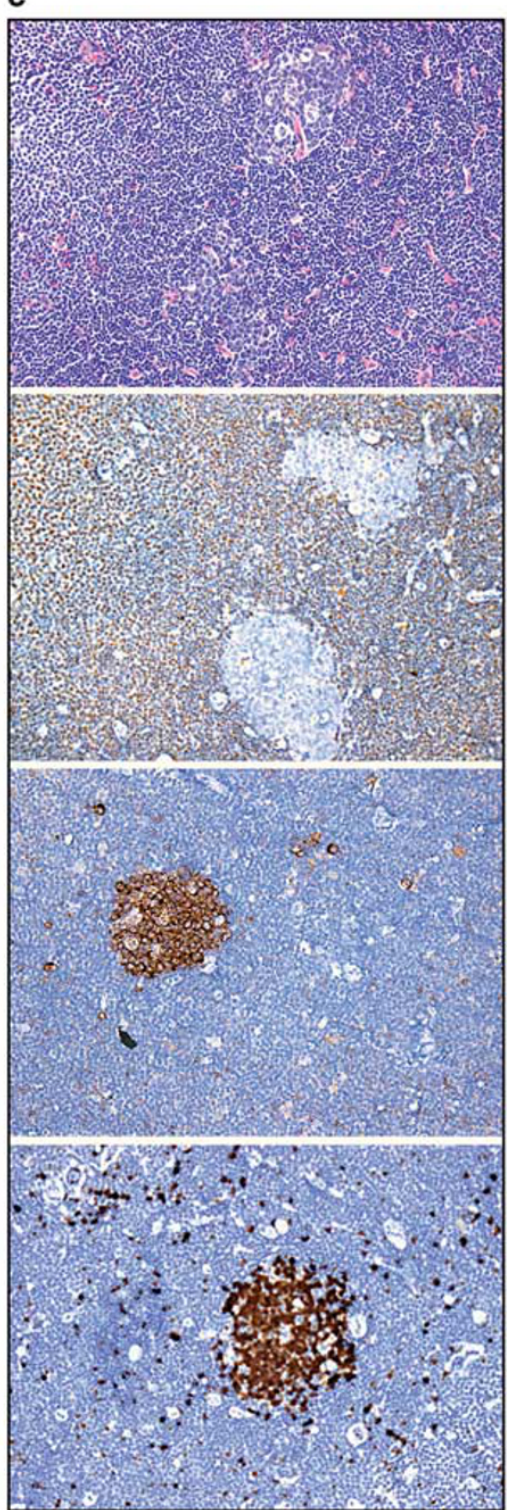

Figure 2 IGF2BP3 protein expression in primary mantle cell lymphomas. IGF2BP3 protein expression in primary mantle cell lymphoma (pilot series) measured by immunohistochemistry. The panels show HE staining, and results for CyclinD1, IGF2BP3 and Ki-67 in a case with high (a), intermediate (b) and low (c) IGF2BP3 protein expression $(\times 200)$.

lymphoma tumors exceeded that of the residual germinal center B cells (Figure 2a).

When we applied the two different scoring systems for IGF2BP3 expression adopted from King et $a l^{19}$ and Schaeffer et $a l,{ }^{15}$ we saw that in the vast majority of the tumor samples the IGF2BP3 expression intensity was associated with the percentage of positively stained tumor cells. In general, mantle cell lymphoma with IGF2BP3 expression in nearly all tumor cells also showed the strongest IGF2BP3staining intensity, leading to very similar results for both scoring approaches. In this pilot series, high IGF2BP3 expression correlated significantly with increased proliferation of the tumor cells measured by Ki-67 using both scoring systems $(P<0.0001)$, as visualized in Figure 2 and summarized in Figure 3a.
Given that increased proliferation of the tumor cells has been shown to be associated with inferior outcome in mantle cell lymphoma patients in several studies, ${ }^{6,22,23}$ we next attempted to correlate IGF2BP3 expression with morphological features of the tumors, the genetic status of $7 \mathrm{p}$ and clinical survival in a larger series of 106 mantle cell lymphoma cases. As already observed in the pilot series, IGF2BP3 protein expression was heterogeneous, and higher expression was strongly associated with increased proliferation measured by the Ki-67 index $(P<0.0001$, Figure 3b). Moreover, increased IGF2BP3 expression was highly correlated with the feature of blastoid morphology $(P=0.003)$. In 34 mantle cell lymphoma tumors, we had the opportunity to compare IGF2BP3 protein expression 

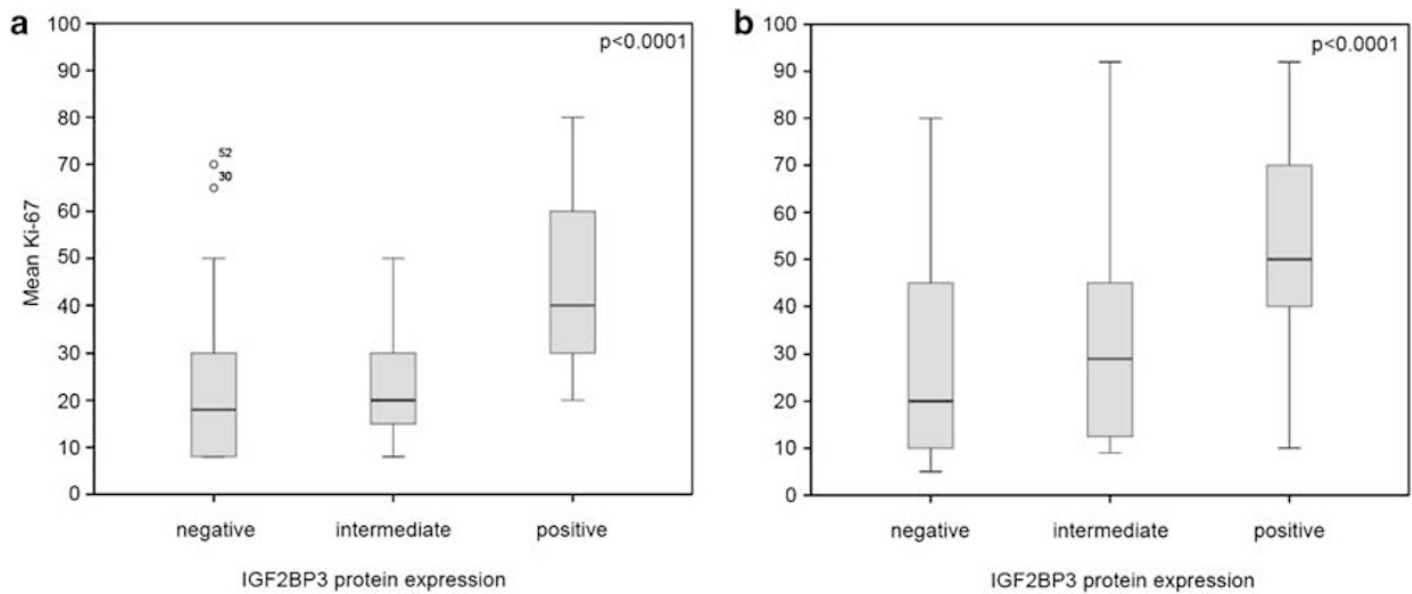

Figure 3 Association of IGF2BP3 protein expression with proliferation. Boxplots depicting Ki-67 level according to the IGF2BP3 protein expression groups in (a) the pilot series and (b) second series. IGF2BP3 scoring adopted from King et al. ${ }^{19}$

with the genomic status of $7 \mathrm{p}$ where the IGF2BP3 gene is located. The chromosomal gain in $7 \mathrm{p}$ is one of the most frequently detected gains in mantle cell lymphoma, associated with an increased proliferation signature and blastoid morphology ${ }^{1,4,5}$ and; in our previous study, ${ }^{5}$ IGF2BP3 mRNA was the most highly and significantly upregulated gene in mantle cell lymphoma tumors harboring a chromosomal gain in $7 \mathrm{p}$. In this study, mantle cell lymphoma with a gain of $7 p$ showed a tendency toward a higher expression of IGF2BP3. However, this association was not statistically significant $(P=0.192$; Figure 4$)$. Despite the fact that in our current series IGF2BP3 expression in mantle cell lymphoma was strongly associated with the Ki-67 index and that Ki-67 expression was strongly correlated with survival $(P<0.0001$, Figure 5a), we were surprised that IGF2BP3 protein expression was not significantly associated with survival in this series $(P=0.335$; Figure 5b).

\section{IGF2BP3 Protein Expression in Mantle Cell Lymphoma at Diagnosis and Relapse}

As protein markers in tumors may exhibit varying expression during the course of the disease, we were next interested to investigate whether IGF2BP3 protein expression may differ in mantle cell lymphoma tumors between primary diagnosis and relapse. Thus, we studied whole tissue sections from seven mantle cell lymphoma samples at both time points. Three mantle cell lymphomas showed a classic morphology in the initial sample as well as in the relapse setting, three mantle cell lymphomas were blastoid at both time points and one mantle cell lymphoma progressed from classic to blastoid morphology at relapse. In these paired mantle cell lymphoma samples, IGF2BP3 expression ranged between 10 and $80 \%$ of tumor cells and

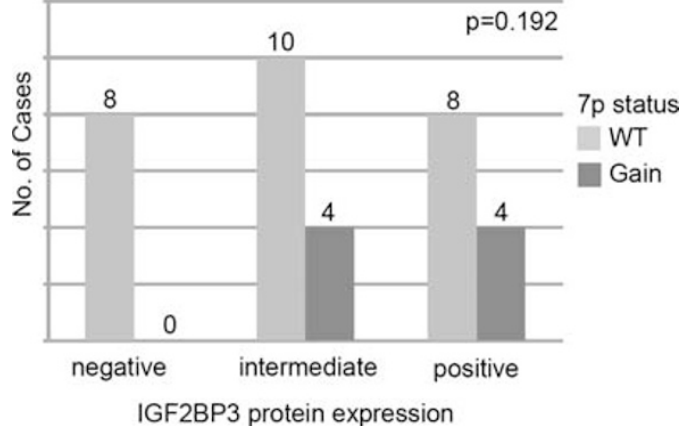

Figure 4 Association of IGF2BP3 protein expression with the chromosomal status of 7p. IGF2BP3 protein expression in a series of 34 primary mantle cell lymphoma (series 2) with available information about the chromosomal status of $7 \mathrm{p}$ according to chromosomal gains of $7 p$ or wild type (WT). IGF2BP3 scoring adopted from King et al. ${ }^{19}$

remained highly constant between primary diagnosis and relapse.

\section{IGF2BP3 Protein Expression in Small Lymphocytic Lymphoma}

As mantle cell lymphomas and chronic lymphocytic leukemias/small lymphocytic lymphomas may show some related morphological and biological features, we finally investigated the IGF2BP3 protein expression in a series of 20 small lymphocytic lymphomas. In contrast to the findings in mantle cell lymphoma that showed a broad range of IGF2BP3 expression, IGF2BP3 staining was overall weaker in small lymphocytic lymphoma (Table 3). Only one small lymphocytic lymphoma was considered positive ( $>50 \%$ of the tumor cells stained), whereas five small lymphocytic lymphoma showed intermediate staining $(10-50 \%)$. A total of 14 small lymphocytic lymphoma were scored negative for IGF2BP3 staining $(<10 \%)$. Interestingly, small lymphocytic lymphoma with prominent proliferation centers often 
a

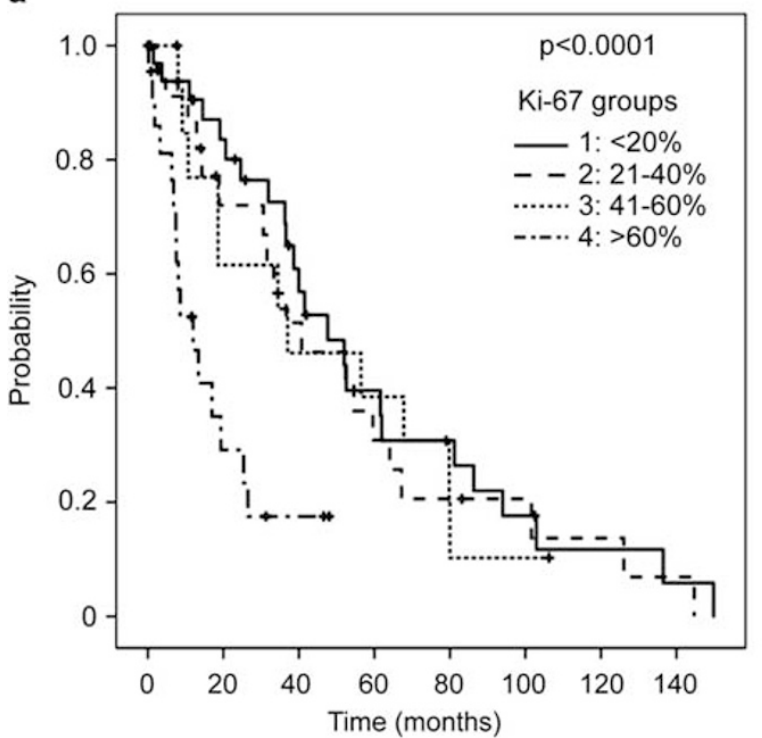

b

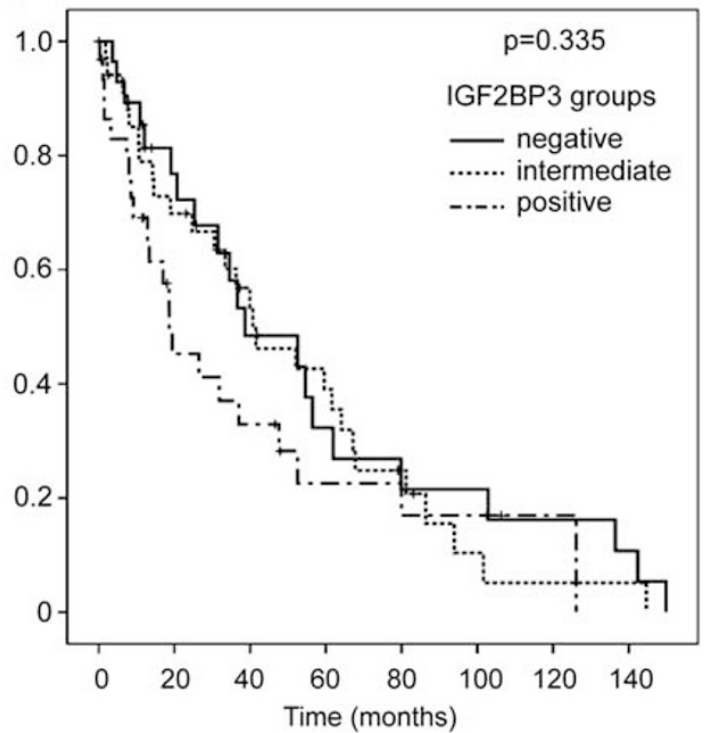

Figure 5 Association of IGF2BP3 protein expression with survival. Kaplan-Meyer plots visualizing survival groups according to (a) proliferation measured by Ki-67 immunohistochemistry (scoring according to ${ }^{22}$ )and (b) IGF2BP3 protein expression measured by immunohistochemistry. IGF2BP3 scoring adopted from King et al. ${ }^{19}$

Table 3 Immunohistochemical expression of IGF2BP3 in primary MCL cases and in CLL. IGF2BP3 scoring adopted from King et al ${ }^{19}$

\begin{tabular}{|c|c|c|c|c|c|c|c|}
\hline & \multirow[t]{2}{*}{ Total no. } & \multirow{2}{*}{$\begin{array}{c}\text { Total } \\
\text { positive }(>50 \% \\
\text { tumor cells) }\end{array}$} & \multicolumn{3}{|c|}{$\begin{array}{l}\text { IGF2BP3-staining intensity } \\
\text { in cases }>50 \% \text { positive tumor cells }\end{array}$} & \multirow[t]{2}{*}{$\begin{array}{c}\text { Intermediate } \\
\text { (10-50\% tumor cells) }\end{array}$} & \multirow[t]{2}{*}{$\begin{array}{c}\text { Negative } \\
(<10 \% \text { tumor cells })\end{array}$} \\
\hline & & & Weak & Moderate & Strong & & \\
\hline Pilot MCL series & 66 & $28(42 \%)$ & 0 & 8 & 20 & $17(26 \%)$ & $21(32 \%)$ \\
\hline Second MCL series & 106 & $38(36 \%)$ & 0 & 2 & 36 & $36(34 \%)$ & $32(30 \%)$ \\
\hline SLL series & 20 & $1(5 \%)$ & 0 & 0 & 1 & $5(25 \%)$ & $14(70 \%)$ \\
\hline
\end{tabular}

Abbreviations: MCL, mantle cell lymphoma; SLL, small lymphocytic lymphoma.

showed an accumulation of IGF2BP3-positive cells in the proliferation centers. Morphologically, these cells corresponded mostly to paraimmunoblasts. No association could be detected between the level of IGF2BP3 expression and the proliferation fraction (Ki-67) in small lymphocytic lymphoma.

\section{Discussion}

Our study demonstrates that IGF2BP3 protein is variably expressed in mantle cell lymphoma and that strong expression in a high percentage of tumor cells is tightly associated with an increased proliferation capacity of the tumor cells. Our previous gene expression and SNP array analysis in mantle cell lymphoma had identified IGF2BP3 as the most strongly upregulated mRNA in mantle cell lymphoma cases harboring chromosomal gains in the region $7 \mathrm{p}$ where the IGF2BP3 gene is located. ${ }^{5}$ The newly generated genomic and gene expression data in eight mantle cell lymphoma cell lines in this study show elevated IGF2BP3 mRNA expression in those cell lines that carry alterations in $7 \mathrm{p}$. This holds also true for primary mantle cell lymphoma tumors in which all cases with a copy number gain of $7 \mathrm{p}$ had increased expression levels of IGF2BP3 (Figure 1), but several mantle cell lymphoma cell lines and primary mantle cell lymphomas without a $7 p$ gain also displayed increased IGF2BP3 mRNA expression. In a series of 45 primary tumors, the IGF2BP 3 protein status determined by immunohistochemistry and the $7 \mathrm{p}$ status showed no significant statistical correlation. However, there was a tendency in mantle cell lymphoma with gains in $7 p$ toward higher IGF2BP3 expression and all mantle cell lymphoma with low or absent IGF2BP3 protein expression lacked copy number gains of $7 p$ (Figure 4). Thus, IGF2BP3 expression in mantle cell lymphoma is obviously also regulated by mechanisms other than the chromosomal copy number status of $7 \mathrm{p}$.

It was previously reported that IGF2BP3 protein expression is a marker of physiological germinal 
center B cells, ${ }^{19}$ which was confirmed in our study. Accordingly, residual germinal centers in lymph nodes with infiltrations by mantle cell lymphoma stained strongly positive for IGF2BP3. An immunohistochemical overview in a limited number of cases from various B- and T-cell lymphoma subsets demonstrated highest expression of IGF2BP3 in Hodgkin lymphoma, diffuse large B-cell lymphoma, Burkitt lymphoma and follicular lymphoma leading to the hypothesis that IGF2BP3 expression might be associated with a germinal center-derived origin of the lymphomas. Only 10 mantle cell lymphoma cases had been studied in that series, most of which showed low or absent IGF2BP3 expression. ${ }^{19}$ In contrast, we here identify a significant subset of mantle cell lymphoma, comprising more than one third of all cases, with strong IGF2BP3 protein expression in a high percentage of tumor cells. Given that mantle cell lymphoma is a non-germinal center-related lymphoma and in view of the tight association between elevated IGF2BP3 expression and increased proliferation of the tumor cells evident in our series, we would like to suggest that IGF2BP3 expression in B-cell lymphomas might be more closely tied to the proliferative capacity of the tumor cells. This idea would be supported by the fact that follicular lymphomas and Burkitt lymphomas, representing typical germinal center-related lymphomas, showed divergent IGF2BP3-staining intensities, with Burkitt lymphomas, in which almost all neoplastic cells proliferate, displaying stronger IGF2BP3 labeling compared with follicular lymphomas, in which the proliferation is much lower. ${ }^{19}$ The link between IGF2BP3 expression and proliferation might be substantiated by our results from the analysis of 20 small lymphocytic lymphoma/chronic lymphocytic leukemia cases, in which the IGF2BP3 protein expression was much lower compared with mantle cell lymphoma. Interestingly, IGF2BP3 expression in small lymphocytic lymphoma/chronic lymphocytic leukemia was found to be pronounced in the proliferation centers, particularly in the paraimmunoblasts, that are supposed to represent the proliferative compartment of chronic lymphocytic leukemia in the lymph node. ${ }^{24}$

There is good evidence from the published literature that the IGF pathway has a significant role in the biological behavior of malignant tumors. ${ }^{10,25}$ Specifically, increased protein expression of IGF2BP3 has been associated with enhanced tumor aggressiveness, metastatic behavior and inferior outcome of various solid cancers. ${ }^{12-18}$ Recently, there has been growing interest in the IGF pathway from a therapeutic perspective, as monoclonal antibodies and small molecules targeting the IGF1 receptor have become available a few years ago and initial, small phase I and II, trials showed encouraging results in individual patients..$^{25}$ In this study, we investigated the mRNA expression levels of several molecules involved in IFG signaling in mantle cell lymphoma cell lines and primary mantle cell lymphoma cases. It became evident that the majority of mantle cell lymphoma cell lines and primary tumors show increased IGF2BP3 mRNA expression, in line with IGF2BP3 belonging to the 'mantle cell lymphoma gene expression signature' as reported in a global gene expression profiling study. ${ }^{6}$ To a lesser extent and more pronounced in some mantle cell lymphoma cell lines, we found that the IGF 1 and 2 receptors are also expressed on the mRNA level, in concordance with a recent study by Vishwamitra et al. ${ }^{26}$ These authors demonstrated elegantly that inhibiting the IGF1 receptor by picropodophyllin or short interfering RNA led to decreased viability and proliferation of mantle cell lymphoma cell lines and primary mantle cell lymphoma cells by inducing apoptosis and cell cycle arrest. ${ }^{26}$ The rate of tumor cell proliferation has been established as a key biological feature of clinical aggressiveness in mantle cell lymphoma, and several genetic events occurring during the progression of the disease appear to enhance proliferation leading to decreased survival times. ${ }^{1}$ Despite the strong statistical association in our cohort between IGF2BP3 protein expression and an increased Ki-67 index of the tumor cells, the major prognostic marker for survival in previous studies, ${ }^{22,23}$ IGF2BP3 expression itself did not correlate with clinical outcome in our analysis reinforcing the idea that the quantitative measurement of tumor cell proliferation in mantle cell lymphoma serves as an integrator of various unfavorable molecular events. ${ }^{6}$

Our finding that IGF2BP3 protein expression is significantly increased in the mantle cell lymphoma subset with elevated tumor cell proliferation adds to the notion that increased IGF signaling in mantle cell lymphoma might contribute additional growth stimuli that enhance the proliferative capacity. Therefore, therapeutic intervention with this pathway, eg, by inhibiting the IGF1 receptor, may represent a viable future treatment option in mantle cell lymphoma. ${ }^{26}$ In this context, it is worthwhile to note that IGF2BP3 protein expression and, hypothetically, the level of IGF signaling appear to be relatively constant in mantle cell lymphoma cells over time, as IGF2BP3 expression was not markedly different in paired mantle cell lymphoma specimens investigated at primary diagnosis and at the time of relapse.

\section{Acknowledgement}

We thank Sabine Roth for expert technical assistance.

\section{Disclosure/conflict of interest}

The authors declare no conflict of interest. 


\section{References}

1 Jares P, Colomer D, Campo E. Genetic and molecular pathogenesis of mantle cell lymphoma: perspectives for new targeted therapeutics. Nat Rev Cancer 2007;7:750-762.

2 Royo C, Salaverria I, Hartmann EM, et al. The complex landscape of genetic alterations in mantle cell lymphoma. Semin Cancer Biol 2011;21:322-334.

3 Allen JE, Hough RE, Goepel JR, et al. Identification of novel regions of amplification and deletion within mantle cell lymphoma DNA by comparative genomic hybridization. Br J Haematol 2002;116:291-298.

4 Bea S, Ribas M, Hernandez JM, et al. Increased number of chromosomal imbalances and high-level DNA amplifications in mantle cell lymphoma are associated with blastoid variants. Blood 1999;93:4365-4374.

5 Hartmann EM, Campo E, Wright G, et al. Pathway discovery in mantle cell lymphoma by integrated analysis of high-resolution gene expression and copy number profiling. Blood 2010;116:953-961.

6 Rosenwald A, Wright G, Wiestner A, et al. The proliferation gene expression signature is a quantitative integrator of oncogenic events that predicts survival in mantle cell lymphoma. Cancer Cell 2003;3: 185-197.

7 Blenk S, Engelmann JC, Pinkert S, et al. Explorative data analysis of MCL reveals gene expression networks implicated in survival and prognosis supported by explorative CGH analysis. BMC Cancer 2008;8:106.

8 Nielsen J, Christiansen J, Lykke-Andersen J, et al. A family of insulin-like growth factor II mRNA-binding proteins represses translation in late development. Mol Cell Biol 1999;19:1262-1270.

9 Yaniv K, Yisraeli JK. The involvement of a conserved family of RNA binding proteins in embryonic development and carcinogenesis. Gene 2002;287:49-54.

10 Pollak M. Insulin and insulin-like growth factor signalling in neoplasia. Nat Rev Cancer 2008;8:915-928.

11 Mueller-Pillasch F, Lacher U, Wallrapp C, et al. Cloning of a gene highly overexpressed in cancer coding for a novel $\mathrm{KH}$-domain containing protein. Oncogene 1997;14:2729-2733.

12 Findeis-Hosey JJ, Yang Q, Spaulding BO, et al. IGF2BP3 expression is correlated with histologic grade of lung adenocarcinoma. Hum Pathol 2010;41:477-484.

$13 \mathrm{Li} \mathrm{D}$, Yan D, Tang $\mathrm{H}$, et al. IGF2BP3 is a novel prognostic marker that correlates with colon cancer progression and pathogenesis. Ann Surg Oncol 2009;16:3499-3506.

14 Hoffmann NE, Sheinin Y, Lohse CM, et al. External validation of IGF2BP3 expression as an independent prognostic marker for metastatic progression and death for patients with clear cell renal cell carcinoma. J Urol 2008;179:133-134.

15 Schaeffer DF, Owen DR, Lim HJ, et al. Insulin-like growth factor 2 mRNA binding protein 3 (IGF2BP3) overexpression in pancreatic ductal adenocarcinoma correlates with poor survival. BMC Cancer 2010;10:59.

16 Jeng YM, Chang CC, Hu FC, et al. RNA-binding protein insulin-like growth factor II mRNA-binding protein 3 expression promotes tumor invasion and predicts early recurrence and poor prognosis in hepatocellular carcinoma. Hepatology 2008;48:1118-1127.

17 Chen ST, Jeng YM, Chang CC, et al. Insulin-like growth factor II mRNA-binding protein 3 expression predicts unfavorable prognosis in patients with neuroblastoma. Cancer Sci 2011;102:2191-2198.

18 Kobel M, Xu H, Bourne PA, et al. IGF2BP3 (IGF2BP3) expression is a marker of unfavorable prognosis in ovarian carcinoma of clear cell subtype. Mod Pathol 2009;22:469-475.

19 King RL, Pasha T, Roullet MR, et al. IMP-3 is differentially expressed in normal and neoplastic lymphoid tissue. Hum Pathol 2009;40:1699-1705.

20 Bea S, Salaverria I, Armengol L, et al. Uniparental disomies, homozygous deletions, amplifications, and target genes in mantle cell lymphoma revealed by integrative high-resolution whole-genome profiling. Blood 2009;113:3059-3069.

21 Swerdlow SHCE, Harris NL, Jaffe ES, et al. (ed). WHO Classification of Tumors of Haematopoietic and Lymphoid Tissues. IARC: Lyon, 2008.

22 Katzenberger T, Petzoldt C, Holler S, et al. The Ki67 proliferation index is a quantitative indicator of clinical risk in mantle cell lymphoma. Blood 2006;107: 3407.

23 Determann O, Hoster E, Ott G, et al. Ki-67 predicts outcome in advanced-stage mantle cell lymphoma patients treated with anti-CD20 immunochemotherapy: results from randomized trials of the European MCL Network and the German Low Grade Lymphoma Study Group. Blood 2008;111:2385-2387.

24 Ponzoni M, Doglioni C, Caligaris-Cappio F. Chronic lymphocytic leukemia: the pathologist's view of lymph node microenvironment. Semin Diagn Pathol 2011;28: 161-166.

25 Maki RG. Small is beautiful: insulin-like growth factors and their role in growth, development, and cancer. J Clin Oncol 2010;28:4985-4995.

26 Vishwamitra D, Shi P, Wilson D, et al. Expression and effects of inhibition of type I insulin-like growth factor receptor tyrosine kinase in mantle cell lymphoma. Haematologica 2011;96:871-880. 